\title{
PSICOLOGÍA EDUCACIONAL DE LAS MATEMÁTICAS
}

\section{Raúl González Moreyra ${ }^{1}$}

Se ha realizado una somera revisión bibliográfica actual para identificar el estado del conocimiento psicopedagógico de la enseñanza-aprendizaje de las matemáticas. Hemos encontrado tres temáticas definidas: la primera sobre el desarrollo cognitivo de los conceptos matemáticos, la segunda sobre los procedimientos de cálculo y solución de problemas de los estudiantes y la tercera sobre las estrategias psicodidácticas de aplicación escolar. En lo que respecta al primer rubro hemos revisado dos áreas, la del desarrollo temprano (cero a 4-5 años) y la del desarrollo operatorio (posterior). Hemos considerado los nuevos hallazgos en lo que hace al número en el niño preverbal y mantenido los aportes piagetanos a partir de las etapas preconservantes. En lo que respecta al segundo tema hemos revisado los errores y precisión del cálculo, las habilidades del cálculo eficaz y el papel de la memoria; además, aspectos de la solución de problemas como la organización del conocimiento del procesamiento cognitivo y la actividad resolutoria. En el tercer tema, que dividimos en psicodidácticas numéricas y conjuntistas, hemos revisado por un lado los aportes de Thordnike, Gagné y Resnick y por el otro los de la Gestalt, Bruner y Dienes. El saldo final es el de un positivo avance en conocimientos y procedimientos sobre psicopedagogía de las matemáticas.

Palabras clave: Enseñanza-aprendizaje, desarrollo cognitivo, estrategias psicodidácticas, conceptos matemáticos, procedimiento de cálculo, solución de problemas.

It was made a brief lo dale bibliographic revision to identify the state of psychopedagogic knowledge of mathematics leaching-learning. We found three defined thematics: the first about cognitive development of the mathematics concepts, the

\footnotetext{
1 Profesor emérito de la UNMSM.
} 
second about the process of calculating and solving of problems by the students and the third about the psycho-pedagogic strategies for school use. Respect the first theme we have reviewed two areas, early development (zero to 4-5 years) and operant development (post). We have considered the new findings in what is made on number in the pre-verbal child and maintained the piagetian ideas from the pre-conserving stages. About the second we have reviewed mistakes and precision on calculating, the abilities of accurate calculation and the function of memo,-y; also, aspects of problem solving as organization of knowledge of the cognitive process and the resolutory activity. In the third theme, which we divided in numerical psycho-didactics and conjunctivas, we have reviewed on one side the ideas of Thordnike, Gagné and Resnick and on the other from the Gestalt, Bruner y Dienes. The final balance is of a positive advance in knowledge and procedures about mathematics psycho-pedagogy.

Keywords : Teaching-learning, cognitive envelopment, strategy psychodidactic, mathematical concept, procedure of calculs, solution of problems.

Uno de los problemas centrales de la educación para el desarrollo y la modernización es el que presenta la enseñanza de las matemáticas en la escuela. La formación de profesores, tanto los de nivel primario como los de nivel secundario, exige plantearse claramente las demandas de competencias que se les deberá exigir durante sus estudios académicos respectivos, a los primeros en su formación general como docentes de ciclo básico, a los segundos como profesores especializados en el área. Deberán ellos, también, elaborar el currículo pertinente para la escolaridad, y definirse en él objetivos, contenidos, metodologías, secuencias, medios y evaluación pedagógica. Deberá, posteriormente, investigarse el impacto social del currículo sobre las competencias matemáticas que los sujetos desplieguen al salir de la escuela después de su exposición sistemática y continua al entorno pedagógico. Tenemos, pues, que definir la formación del docente, elaborar y aplicar currículos y averiguar el impacto o efecto de estas experiencias sobre la población afectada. Se requiere para responder eficazmente a estas tareas tener una idea clara del estado actual de la pedagogía de la matemática. Un insumo importante para este conocimiento 
son los avances que recientemente han estado haciéndose en la Psicología Cognitiva aplicada a la educación matemática. En ella se han afrontado tres problemas centrales: el del desarrollo, el de los mecanismos cognitivos involucrados en el procesamiento matemático y el de la enseñanza-aprendizaje.

El problema del desarrollo no es otro que el problema de la evolución ontogenética del pensamiento matemático. Los aportes centrales a este respecto son sin dudarlo los aportes piagetanos, nosotros los tendremos en cuenta, pero además, daremos mucha importancia a aportes más recientes vinculados a nuevas concepciones sobre el desarrollo temprano de aspectos esenciales del pensamiento matemático como el número, el conteo y la subitización. Expondremos en dos partes los problemas del desarrollo: una primera parte dedicada al desarrollo temprano centrada en los avances más recientes hechos sobre los procesos de pensamiento matemático del niño pequeño. Una segunda parte la dedicaremos al desarrollo operatorio, con gran énfasis en la concepción de Piaget sobre el desarrollo de las matemáticas en el niño y el adolescente.

El problema de los mecanismos implicados en la cognición matemática lo enfrentaremos bajo dos rubros: procedimientos de cálculo y de resolución de problemas, que visualizan la dicotomía entre los procedimientos algorítmicos y procedimientos heurísticos. En los primeros existen reglas de cómputo que llevan a un resultado final, en los segundos los problemas tienen vías más difusas que deberán identificarse y, que no siempre llevan a una solución final, sino que a veces sólo a aproximaciones.

Nuestro tercer problema, el de la enseñanza-aprendizaje, también cubrirá dos aspectos generados por dos enfoques distintos a las preguntas complementarias sobre cómo aprende matemáticas el niño y cómo debe enseñársele. El primer enfoque se orienta hacia estrategias algorítmicas y numéricas que privilegian el dominio del cálculo. El segundo enfoque se orienta hacia las estructuras matemáticas, los conjuntos, los dispositivos lógicos y algebraicos subyacentes a todas las competencias del área. Es una orientación vinculada a los desarrollos de la matemática moderna. 


\section{DESARROLLO TEMPRANO}

\subsection{Detección numérica}

Las investigaciones más recientes en el niño de cero a cuatro años han revelado la presencia de capacidades cognitivas en general, y matemáticas en particular, mayores y más complejas de las que se presumía poseía hasta hace pocos años. Bajo el rubro de detección numérica veremos los conceptos de numerosidad, zoonumerosidad, subitización y conteo, y desarrollaremos algunos aspectos relevantes de investigaciones pertinentes.

\subsection{Numerosidad}

El trabajo paradigmático se debe a los experimentos de Antell y Keating. Se les presentó a niños recién nacidos a través del procedimiento habituación y deshabituación tarjetas que contenían el mismo número de puntos, pero variando en longitud de líneas y densidad de puntos. Luego se les mostraba una tarjeta posthabituación con un número distinto de puntos (se mantenía longitud y densidad). Al cambiar el número emergía el proceso de atención renovada. Si se sustituían los puntos por objetos de diferentes colores y formas se obtenían los mismos resultados. En otros experimentos con niños algo mayores de seis a ocho meses, se logró igual detección de numerosidad pero con carácter interno sensorial (auditivovisual).

\subsubsection{Subitización}

Se define a la subitización como el reconocimiento inmediato sin conteo explícito de un conjunto pequeño de objetos. Las formaciones de hasta tres a cuatro puntos se subitizan fácilmente. Si se incrementan los puntos a seis o diez ya no es posible resolver la tarea. En los adultos el costo en términos de tiempo es de 50 milisegundos por cada cifra adicional, hasta cuatro cifras en experimentos con niños de cinco años. En niños menores de seis a ocho meses alcanza, según Gelman, hasta 
tres elementos, sugiriéndose la existencia de predisposiciones innatas que influyen sobre la atención y la memoria, lo que permitiría el almacenamiento de las representaciones numéricas. No se iniciaría el desarrollo cognitivo del niño con vagas distinciones "muchos-pocos", sino habrían tempranamente procesos numéricos con características de dominio específico.

Se han propuesto dos teorías principales para explicar este proceso de subitización. Una teoría es la de Glasersfeld, de base perceptiva y sin procedimientos numéricos. Gallistel y Gelman han propuesto por su parte una teoría de recuento rápido generada por una predisposición de dominio específico sin base perceptiva.

\subsubsection{Conteo}

Gelman ha propuesto cinco principios del aprendizaje del conteo que funcionan como reglas de predisposición innatas.

I. Principios de correspondencia biunívoca: un elemento de una colección con uno de la otra.

II. Principio de ordenación estable: el recuento es independiente de los rótulos que se unen, como por ejemplo cuando se aplica 1, 2, 4, pero no se repite ningún rótulo.

III. Principio de indiferencia de elementos: puede contarse cualquier clase de objetos.

IV. Principio de indiferencia de orden: el conteo es en cualquier secuencia.

V. Principio de cardinalidad simple: el último término del recuento da el valor cardinal del conjunto.

Desde el punto de vista operacional, el conteo tiene las siguientes propiedades: 
a) Demora en los adultos $1 / 3$ de segundo por objeto, en los niños demora $2 / 3$ de segundo también por cada objeto.

b) Los matemáticos hábiles empiezan a usar desde los cuatro años para contar conjuntos grandes, el agrupamiento en subconjuntos. Se cuenta cada subconjunto y se acumulan al final. Las pistas como marcas, colores y distancias, favorecen los agrupamientos. Si las pistas están distribuidas al azar no se producen los agrupamientos.

c) El conteo de objetos es más difícil cuando estos están fijos y dispersos; lo es menos cuando están fijos alineados; y aun menos difícil cuando son móviles y pueden agruparse una vez contados.

d) El principio de cardinalidad parece actuar según Wynn sólo a partir de los tres años, los niños menores aun cuando puedan contar bien hasta cinco, cuando se les pregunta por cuánto hay, no dan la última cifra como respuesta.

Finalmente debe señalarse que está firmemente establecida la capacidad animal para discriminar tres a cuatro objetos. Algunas especies discriminan entre cantidades grandes (40-50 picotazos).

Gallistel ha propuesto como mecanismos los principios de indiferencia de elementos y correspondencia biunívoca. En esto los animales se asemejan al niño pequeño, pero no en el principio de indiferencia del orden, su recuento es unidireccional. El niño tiene mayor complejidad de principios y la potencialidad para pasar a un sistema simbólico de exclusividad específica.

\subsection{Desarrollo simbólico}

La detección temprana de la numerosidad y el conteo son el inicio de un proceso cuyo primer desarrollo deberá culminar en la adquisición y uso de los sistemas simbólicos (lenguaje) y conceptuales (teorías) pertinentes que le servirán de base a la expansión futura de sus competencias matemáticas. Los temas de estos acápites serán los numerales, la notación y la matemática infantiles. 


\subsubsection{Numerales}

Los numerales se definen como los términos del lenguaje oral que rotulan a los números en cuanto cantidades. Los numerales se aprenden por reglas diferentes al etiquetado de objetos o denominación. La base de este aprendizaje son los principios de irrelevancia del objeto y de ordenación estable, que no son aplicables para nombrar objetos. Ambos dominios no se confunden ante la masa de estimulación, debido según Gelman a la influencia de los principios innatos de dominio específico.

A la importancia del léxico numérico debe añadirse la importancia del aprendizaje del lenguaje matemático en general (léxico y gramática) y a hacerlo independientemente del lenguaje estándar cotidiano. Los buenos matemáticos dominan el lenguaje matemático, y este dominio correlaciona positivamente con la comprensión matemática.

\subsubsection{Notación}

La notación numérica es logográfica a diferencia de la escritura que es fonográfica, mientras el dibujo es pictográfico.

El desarrollo cultural y su dominio individual son dos aspectos indispensables para la evolución de la competencia matemática.

Basados en Hugues proponemos un desarrollo de la notación en tres etapas:

Primera etapa: garabato, no se identifica ninguna asociación entre el elemento gráfico y el elemento numérico.

Segunda etapa: biunívoca, en este período correlacionan el número de unidades gráficas con el número de objetos. El grafismo empleado primero es pictográfico (dibujo del objeto) y luego es icónico (signos). Si se le pregunta que diga por escrito ¿cuántos bloques hay? en un grupo de tales que se le proporciona, en la sub-etapa pictográfica los representa con dibujos que semejan los bloques con indicación de la cantidad, forma, posición y orientación de estos. En la sub-etapa icónica dibuja marcas con correspondencias con los bloques y cada signo representa un bloque distinto. 
Tercera etapa: simbólica, se subdivide según Stallard en cuatro subetapas: 1) Dominio de cifras simples; 2) dominio de operaciones sencillas como $2+2=4$ (realización); 3) dominio de operaciones más difíciles como $4 ; 4+0=; i+3=5 ; 4)$ Dominio posicional: uso de cifras mayores como 3,405 , etc.

\subsubsection{Metamatemática}

Se define como metamatemática a las ideas peculiares del niño sobre el número. Hay dos teorías principales de carácter secuencial La primera teoría investigada por Gelman asume los números como lo que se obtiene al contar, el número se piensa como una propiedad de las cosas contables. En esta teoría ni el cero ni las fracciones son números.

Para la segunda teoría el número es algo con lo que se realizan y a los que se aplican operaciones matemáticas. En esta concepción cambia la naturaleza del cero y de las fracciones. Wellamn y Miller investigaron las ideas sobre el cero y señalaron tres estadios de progreso: el primero es de familiarización y notación del cero independientemente de su comprensión; en el segundo estadio comprende que el cero hace referencia a ninguno o nada; en el tercer estadio comprende que es el número más pequeño de la serie de enteros no negativos.

Karminoff-Smith investigó el desarrollo de las ideas del niño sobre las fracciones: primero el niño identifica a las fracciones con el papel que los enteros cumplen en las operaciones (sumará $1 / 2+1 / 4=1 / 6$ ). En una segunda etapa comprende que los dos números implican la división entre números distintos. Se va más allá del número en relación con lo real para definirlo como relación entre números distintos. Luego va más allá del número en relación con lo real para definirlo como pura relación entre números.

\section{DESARROLLO OPERATORIO}

\subsection{Las operaciones intelectuales}

Piaget definió las operaciones como acciones simbólicas, interiorizadas y reversibles. El desarrollo de las operaciones significa: 
a) diferenciación simbólica entre significantes y significados; b) restricciones lógicas al operar conscientemente con los símbolos y c) desarrollo del pensamiento verbal.

Los niveles piagetanos de operaciones intelectuales son:

Etapa preoperatoria (2-7 años): existe la diferenciación significantesignificado, pero sin restricciones lógicas.

Etapa lógico-concreta (7-12 años): operaciones en el plano de las representaciones, estructura el presente en función del pasado sin las deformaciones, dislocaciones ni contradicciones del niño preoperatorio.

Etapa lógico-formal (12-16 años): operaciones sobre lo posible configurando lo real como uno de sus casos. Frente a un problema se preveen todas las relaciones de posible validez y luego se determina por experimentación y análisis lógico cuáles de las relaciones posibles tiene validez real. Es un pensamiento hipotético-deductivo, proposicional y probabilístico.

Según Piaget las principales características de las operaciones son:

- Transformaciones de los símbolos en forma interiorizada y reversible configurando estructuras y sistemas de procesamiento (razonamiento).

- Aprehensión simultánea simbólica e interna de una síntesis única de una serie completa de hechos separados.

- Reflexión sobre la organización de los propios actos: carácter contemplativo, no sólo activo.

- Independización más allá de los actos presentes y de los objetos concretos del entorno real: transición a manipular simbólicamente entidades no tangibles.

\subsection{Nivel preoperatorio o preconservante}

En términos piagetanos es el niño preconservante de cantidad, con dificultades para conservar la cantidad de líquido, las discontinuidades espaciales en correspondencia y otras transformaciones geométricas y 
numéricas. Al final de esta etapa se desarrollan las primeras operaciones intuitivas de conservación de la cualidad y de función orientada.

\subsubsection{Transformaciones numéricas}

Correspondencias biunívocas. Dificultad entre los cuatro a seis años para poner en correspondencia objetos como flores y floreros, verificándola al poner la flor dentro del florero. Los pequeños la descubren por la relación contenido-continente.

Relaciones de equivalencia. Dificultad para establecer la correspondencia con F1 (flores del experimento del párrafo anterior) con F2 (un nuevo conjunto de flores), después que se ha trabajado sólo con F2 igual F" pero de diferente clase y en los mismos floreros.

Adición de clases. Experimentos en los cuales se muestra un conjunto de bolas de madera con más bolas rojas que blancas. Dificultad para responder si hay más bolas rojas o de madera.

Composición aditiva $(4+4=7+1)$ : Experimentos del tipo: si un día come cuatro galletas en la mañana y cuatro en la tarde, al día siguiente le dan igual cuatro, para la mañana y cuatro para la tarde; pero no tiene hambre y deja tres para la tarde; ¿ha comido igual los dos días?

Correspondencia ordinal. Diez muñecos y diez bastones de tamaño graduado de mayor a menor en desorden, debe darle a cada muñeco el bastón suyo para que pueda salir a pasear. Comparación global del niño, sin seriación ni correspondencia biunívoca.

Conservación de la cantidad. Esta puede ser continua o discontinua. Se explora por correspondencias entre el contenido de una botella y en contenido vaciado en vasos uno alto y el otro ancho.

\subsubsection{Transformaciones geométricas}

Construcción de la medida. Construir una torre igual a otra de $80 \mathrm{cms}$. Hecha de cubos, sobre una mesa casi un metro más baja que la del modelo. Fracasa, su copia es sumaria, imperfecta y sin análisis. 
Conservación de la longitud. Si bien son buenos perceptores al taquitoscopio de segmentos desplazados. cuando a nivel representacional se desplaza una varilla, implica un cambio de evaluación: uno u otro se hace más grande o más chico.

Lugares geométricos. Se le propone materializar en dibujos que elija, la posición de sus camaradas de juego lanzando una pelota a un centro común que esté igual de lejos para todos en un círculo.

Geometría del espacio. Parte de referir todos los puntos del espacio a un punto de vista único y propio. Sobre una maqueta se le pregunta por posiciones próximas o cercanas desde perspectivas diferentes a las propias.

\subsubsection{El problema de la conservación y teorías explicativas}

El problema de la conservación puede plantearse así: ¿cómo los niños muy pequeños que ya poseen numerosidad, cuentan y poseen principios para hacerlo y, además comprenden la cardinalidad, sin embargo fallan en la conservación? Se han elaborado tres teorías importantes:

a) Teoría de Piaget: los niños no tienen un conjunto de principios numéricos explicativos.

b) Teoría de Gelman: falta la representación abstracta (algebraica) de razonar sobre relaciones numéricas sin representación concreta.

c) Teoría de Karmiloff-Smith: se debe a un proceso de redescripción que pasa de la conservación embutida en el recuento a hacerla explícita en la actividad de contar, es decir extraerla para utilizarse con cantidades sin especificar.

\subsection{Nivel lógico concreto}

Se desarrollan de siete a doce años operaciones capaces de manejar representaciones bajo restricciones lógicas. Estas operaciones son las de 
clasificación jerárquicas, seriación ordenada y conservación de invariantes (cantidad, peso y volumen).

\subsubsection{Operaciones aritméticas}

Consisten en el grupo aditivo de números enteros con composición $(1+$ $1=2 ; 2+3=5$; etc. $)$; asociatividad $[(1+1)+1=1+(1+1)]$; inversos $(-1 ;-$ $2)$; identidad (0); iteración $(1+1=2 ; 2+1=3$; etc. $)$.

El otro grupo es el multiplicativo de números enteros con composición $(2 \times 1=2 ; 3 \times 2=6$; etc. $)$, asociatividad $[(1 \times 2) \times 3=1 \times(2 \times 3)]$; inverso ( $(1$; (2); identidad (1).

El grupo es un sistema de transformaciones con composición, asociatividad, reversibilidad (inversión) e identidad. La interacción convierte a los elementos lógicos intensivos en cuantificables.

\subsubsection{Operaciones métricas}

Es la medición de relaciones partes a todo, en la que las partes se convierten en unidades iterables a las que se aplican números. Depende de operaciones infralógicas que son operaciones lógicas aplicadas a los objetos y a las relaciones espacio-temporales de sus partes, en virtud de lo cual: 1) el objeto es una entidad única, sus partes no permanecen ni independientes ni separadas cuando componen un todo; 2) la constitución del objeto requiere proximidad de sus partes; 3) sus operaciones constitutivas son síntesis y partición que se corresponden con adición y sustracción de clases. En las operaciones métricas se aplica la lógica de la cuantificación al mundo físico. Los niños avanzan en la medición usando primero su cuerpo como medida común, para luego usar objetos independientes.

\subsection{Nivel lógico formal}

Las operaciones concretas tienen, como punto de partida lo real.

Están ligadas al aquí-ahora, son como "islotes funcionales" que no configuran un sistema por el que ante un problema el niño pueda pasar de 
una estructura operatoria a otra. En cambio, las operaciones lógico formales elaboran lo potencial y posible como distinto de lo real; se desarrollan estrategias hipotético-deductivas y las entidades más importantes en el razonamiento no son las representaciones de objetos y hechos concretos sino el pensamiento proposicional, generado a partir de las vinculaciones lógicas entre las proposiciones resultantes de las operaciones concretas. Estos vínculos interproposicionales son implicaciones, conjunciones, disyunciones, identidades, etc. Son operaciones de segundo grado, operaciones sobre operaciones.

Las propiedades del nivel lógico formal en términos piagetanos son la combinatoria intraproposicional que consisten en la red de dieciséis combinaciones posibles dado $\mathrm{A}=$ niños aprobados, $\mathrm{y} \mathrm{B}=$ niñas aprobadas (A; $\mathrm{B} ; \mathrm{AB} ; \mathrm{AB} ; \mathrm{AB}$; etc.). La otra propiedad es la de las transformaciones interproposicionales del grupo INRC (identidad, negativa, recíproca $\mathrm{y}$ correlativa). El sistema aplicado al mundo físico de aumentar viscosidad (I) o disminuir peso (C) para resistir el desplazamiento de un pistón se completan con sus negaciones respectivas: disminuir viscosidad (N) y aumentar pero (R), constituyendo un grupo de transformaciones interpreposicionales.

2.4.1. Esquema de combinaciones El experimento tiene tres etapas:

a) Se proporcionan cuatro frascos semejantes con líquidos inodoros e incoloros, son: 1) ácido sulfúrico diluido; 2) agua; 3) agua oxigenada y además 4) una botella con cuentagotas y con yoduro de potasio.

b) Se le presentan dos vasos al sujeto. El primero tiene $1+3$; el segundo tiene dos. Le echa $g$ al primero $(1+3+g)$ que da un color amarillo. Le echa $\mathrm{g}$ al segundo $(2+\mathrm{g})$ que no cambia de color. El yoduro en $(1+3+\mathrm{g})$ es oxidado por el agua oxigenada en un medio ácido, lo que produce el color. En el segundo $(2+\mathrm{g})$, el agua es neutra y su mezcla no cambia el color. Si se añade d a $(1+3+g)+4$ lo decolora. El niño debe repetir la experiencia de mezclar dando color amarillo. 
c) El sujeto preformal no agota las combinaciones. El sujeto formal las realiza en orden. Ej.: $(2+\mathrm{g}) ;(1+\mathrm{g}) ;(3+\mathrm{g})$ y $(4+\mathrm{g})$. Luego pasa a $(1+2+\mathrm{g})$; $(1+3+g) ;(1+4+g) ;(2+3+g) ;(2+4+g) ;(3+4+g)$. En seguida pasa a probar combinaciones con tres frascos y el grupo total. A pesar del resultado (1 $+3+g$ ), trata de agotar todas las combinaciones para explicar el resultado.

\subsubsection{Esquema de proporciones}

En condiciones de experimentos como los del pistón, peso y viscosidad del líquido se tiene la relación proporcional $\mathrm{I} / \mathrm{R}=\mathrm{C} / \mathrm{N}$. Si $\mathrm{I}=\mathrm{P} ; \mathrm{R}=\mathrm{q} \mathrm{y}$ $\mathrm{N}=\mathrm{p}$; entonces se tiene $\mathrm{p} / \mathrm{q}=\mathrm{q} / \mathrm{p}, \mathrm{Y}$ en consecuencia $\mathrm{p} \vee \mathrm{p}=\mathrm{q} \vee \mathrm{q}$.

\subsubsection{Esquema de correlaciones}

Se plantea el problema de la correlación entre color de ojos y color de cabello, en el que:

$$
\begin{aligned}
& a=\text { ojos azules y cabellos rubios }(p . q) \\
& b=\text { ojos azules y cabellos negros }(p . q) \\
& c=\text { ojos negros y cabellos rubios (p.q) } \\
& d=\text { ojos negros y cabellos negros(p.q) }
\end{aligned}
$$

Sólo si $(a+d)$ es mayor que $(b+c)$ en función de la totalidad de posibilidades existe la correlación, que será mayor cuanto mayor sea la diferencia $a+d>b+c$.

\subsubsection{Esquema de distribución}

Corresponde a la distribución de Gauss a través del experimento de lanzar bolitas por un orificio central a cuatro cajas planas inclinadas, cuya parte inferior se divide en dos, tres, cuatro o 18 casilleros y la parte superior tiene una abertura central en forma de embudo.

El niño debe prever la distribución. El preformal sólo prevee distribución uniforme en general, y nunca la simetría de los casilleros 
externos. Puede predecir el mayor número en el casillero central. El niño formal anticipa distribución en campana y equilibrio en casilleros externos.

\section{PROCEDIMIENTOS DE CÁlCULO}

El cálculo es el conjunto de algoritmos y procedimientos computacionales mediante los que se manipulan los números y sus símbolos.

\subsection{Precisión}

Los errores en el cálculo pueden ser de dos tipos: a) en la tarea meta se aplican procedimientos incorrectos y b) en las subdestrezas prerrequisitos hay falta de habilidades pertinentes. Los errores se evalúan de la siguiente manera:

a) Los errores que sólo se dan en la tarea-meta se deben a procedimientos incorrectos.

b) Los errores que se dan conjuntamente en habilidades previas y tareameta son falta de conocimientos prerrequisitos.

\subsection{Procedimientos incorrectos}

\subsubsection{Los errores como fuente de datos}

Deben verse los errores no como señal de falta de inteligencia, sino como fuente de datos y ayuda para comprender la naturaleza del desempeño. Deben elevarse estos a un nivel metacognitivo generando la conciencia de los errores como producto de una secuencia incorrecta o incompleta; el conocimiento procedimental nuevo generado así será más independiente.

\subsubsection{Errores en la resta (un ejemplo)}

Son de dos tipos: a) inversión de la diferencia cuando se resta siempre de la cifra mayor, ej.: $62-39=37$; y b) el manejo del cero que produce las mayores dificultades, entre ellas: 
a) Tomar prestado de cero: $103-45=158$.

b) Saltar la columna correspondiente para tomar préstamo, tomando de la siguiente: $503-114=299$.

c) $\mathrm{O}-\mathrm{N}=\mathrm{N}$, cuando el dígito de arriba es cero se pone en la diferencia el de abajo $\mathrm{N}$.

d) $\mathrm{O}-\mathrm{N}=\mathrm{O}$, cuando el dígito de arriba es cero se pone en la diferencia cero.

e) $\mathrm{N}-\mathrm{O}=\mathrm{O}$, cuando el dígito de abajo es cero se pone en la diferencia cero.

\subsection{Falta de habilidades previas}

\subsubsection{Análisis de tareas lógicas}

Es preguntarse ante una tarea: qué tiene que saber hacer una persona para resolver correctamente esta clase de tareas suponiendo que sólo se le proporcionan instrucciones. Identificadas las habilidades prerrequisito a ellas también se les puede aplicar un análisis de tarea lógico, avanzando a un segundo nivel de análisis. Los datos empíricos señalan que es muy común que este segundo nivel sea el que falle.

\subsubsection{Habilidades pertinentes, tiempo de aprendizaje y nivel de competencia}

Trabajos de Gagné y Paradise encontraron que la correlación entre las habilidades pertinentes y el tiempo de aprendizaje con el nivel de competencia eran de .78 y .82. Una medida de inteligencia general (vocabulario) tenía una correlación de .18 y .22, con tiempo de aprendizaje y nivel de competencia.

\subsection{Cálculo eficaz}

\subsubsection{Almacén declarativo}

Al inicio del aprendizaje el almacén puede ser declarativo, aún no se 
ha procedimentalizado por lo que el trabajo es lento e impreciso.

\subsubsection{Números de pasos}

En la suma los niños pequeños usan primero iteración (+ 1); la de los mayores recupera información pertinente de la MLP, lo que la hace más eficaz. Hay cinco procedimientos iterativos:
a) Contar ambas cantidades, es el más eficaz.
b) Contar desde el primero sumando.
c) Contar desde el segundo sumando.
d) Contar desde el número menor.
e) Contar desde el número mayor, es el más eficaz.

\subsubsection{Procedimientos compuestos}

Los matemáticos hábiles configuran los diversos pasos de cálculo como un sistema de producción reduciendo el número de ellos. Se encontró en estudiantes expertos un promedio de 2.7 pasos y en los principiantes uno de 3.7 .

\subsubsection{Estimaciones}

Las estimaciones son aproximaciones tentativas a los resultados. su función es el actuar conjuntamente con el cálculo y al llegar con ambos procedimientos a resultados similares, servir de comprobación del cálculo hecho. Las grandes discrepancias (no las pequeñas) señalan error. Las estrategias de estimación son tres:
a) Reformulación, redondear números para hacerlos manejables.
b) Traducción, modificar la estructura del planteo por otra, ej.: $492+504+487=500 \times 3$.
c) Compensación, ajustes en una dirección que se equilibran con ajustes en otra.

\subsection{Papel de la memoria}

\subsubsection{Papel de la memoria de largo plazo}

La memoria de largo plazo cumple tres roles fundamentales: 
- Integración de la representación (Larkin): es como se conectan y relacionan entre sí los conceptos en forma rica y ordenada. Se explora por asociaciones sobre el tema, con ráfagas asociadas a un concepto organizador: el experto produce bloques de ítems, el inexperto los recupera al azar.

- Correspondencia con tema: debe haber un ajuste entre las ideas del sujeto sobre el tema con la estructura del contenido y la estructura de los expertos (libros de texto y enseñanza). Se usan para estimarla métodos de asociación de palabras, agrupación de tarjetas y tests gráficos.

- Grado de conexión: relaciones del tema con otros conocimientos de la persona. Se estiman a partir de actuaciones y entrevistas. Por ejemplo el dominio de la resta implica conexiones con el conocimiento del valor posicional de las cifras.

\subsubsection{Papel de la memoria de corto plazo}

Siegel y Ryan compararon niños con problemas de lectura, con trastornos de atención y con dificultades específicas de matemáticas. Todos tenían dos tareas, una verbal y otra numérica. La primera era decir las palabras que faltaban en frases. La segunda era identificar puntos amarillos, en tarjetas distribuidas al azar, entre puntos azules. Luego de cada tarea debían decir las palabras y los números respectivamente y en el mismo orden. Los niños con dificultades específicas tenían puntuaciones bajas sólo en la tarea numérica y no en la verbal.

\section{RESOLUCIÓN DE PROBLEMAS}

Los problemas matemáticos exigen para ser resueltos "comprensión matemática", es decir saber cuando hay que utilizar un procedimiento u otro para solucionarlo. Es una utilización flexible y apropiada de los sistemas de proposiciones y producciones: este conjunto se denomina "organización del conocimiento".

Según Loftus y Suppes las variables de dificultad de un problema son las siguientes: 

a) Número de operaciones necesarias.
b) Relación de los procedimientos del problema con el anterior.
c) Longitud léxica del enunciado
d) Complejidad gradual del enunciado.
e) Conversión de unidades de medida.

\subsection{Organización del conocimiento}

La organización del conocimiento específico influye en la solución de problemas de un campo dado porque guía la búsqueda de solución. Una solución rápida y precisa depende de la organización del conocimiento (DDC). Esta es como un mapa cognitivo de un área determinada.

\subsubsection{Estructura asociativa}

La ODC de los expertos es muy similar con la estructura aceptada de un área de estudios. Se puede investigar por asociaciones libres a partir de los términos claves del área. Por ejemplo, Gagné estudió las asociaciones libres (5) a los términos sumar, restar, multiplicar y exponente. Los conceptos de suma y resta están más próximos entre sí que con el concepto exponente, pero sumar está más cerca de exponente que restar.

\subsubsection{Asociaciones post-estudio}

Greeslin y Shavelson exploraron las asociaciones antes de estudiar (pretest) y dos semanas después (post-test) y dos semanas después (retención). En el post-test y la retención el grupo experimental alcanzó una ODC similar a la del texto.

\subsubsection{Enunciados narrativos}

Silver pidió a los sujetos ordenar 16 problemas en grupos relacionados matemáticamente. Los problemas tenían dos clases de relaciones: por el contenido al tratar de igual tema como por ejemplo la agricultura; la otra relación era el proceso: se tiliza el mismo procedimiento matemático de 
solución. Luego sobre la base de solución a 12 de esos problemas se dividió a los sujetos en buenos, medios y malos solucionadores. Los buenos clasificaron los problemas casi totalmente por el proceso y los malos los clasificaron con ligero dominio por el contenido. Los medios también los clasificaron por el proceso.

\subsection{Procesamiento cognitivo}

El procesamiento cognitivo implica a qué se le presta atención y qué se recuerda. Atender y recordar son dos aspectos claves del procesamiento cognitivo.

\subsubsection{La atención}

Robinson y Hayes estudiaron la diferenciación de los aspectos relevantes de un problema. Los buenos estudiantes se desempeñaron de la siguiente manera:

- Los segmentos relevantes de los enunciados fueron identificados como tales en mayor proporción que los irrelevantes (90-20\%).

- Si la pregunta se ubica al inicio del enunciado es más probable identificar a la primera lectura los aspectos relevantes, que si se pone al final: la pregunta es orientadora.

- En la segunda lectura sólo mejora la performance en los que habían leído la pregunta al final.

- Aspectos irreleventes no matemáticos en ese contexto son más proclives a la confusión.

\subsubsection{La retención}

Mayer descubrió que los estudiantes recordaban mejor los aspectos relevantes de los problemas que más frecuentemente aparecen en los textos. Los estudiantes recordaran mejor las Proposiciones de asignación (dar un valor numérico a una variable, ej.: el camión partió a las 13 horas) que las proposiciones de relación (describir con un valor numérico la relación entre dos variables, ej. un segundo camión viaja a la velocidad de $15 \mathrm{kms}$. Hora más que el primero. Las proposiciones de asignación son el 
$61 \%$ de los enunciados de los problemas, las proposiciones de relación son el $11 \%$ en los textos. El $29 \%$ de proposiciones de relación se olvidan, frente a sólo el $9 \%$ de preposiciones de asignación.

\subsection{Solución de problemas}

Siguiendo a Resnick estudiaremos cuatro secuencias básicas: representación del problema, datos del problema, instrucciones del problema y soluciones del problema.

\subsubsection{Representación del problema}

El sujeto identifica y codifica las características del problema para hacerlo interpretable por los mecanismos de procesamiento. Forma representaciones estableciendo vínculos entre el planteamiento del problema y su propia red semántica y procedimental (matemática y espacial); participan aquí las habilidades lingüísticas generales, el conocimiento del vocabulario matemático y las imágenes que traducen icónicamente las características del problema.

\subsubsection{Datos del problema}

Se denomina entorno de la tarea pues comprende los elementos disponibles y que son percibidos. Suscitan información específica e inspiran procedimientos determinados. El entorno también puede dar lugar a errores porque los datos orientan mal a la solución. Este proceso se adecua al encarrilamiento o fijación funcional de la Gestalt.

\subsubsection{Instrucciones del problema}

Activan esquemas representacionales en función del modo como se propagan. Katona experimentó con tres instrucciones diversas para recordar un número largo: darlo en las centenas que lo componen (tres cifras), insertarlo como PBI de la nación y proponerlo como una serie numérica. Activó tres tipos de estrategias con diferente eficacia cognitiva. Las instrucciones que facilitan representaciones ricas, 
inspirando el uso de conceptos pre-aprendidos, tiene un efecto diferente al de instrucciones para ejecutar rutinas ejercitadas.

\subsubsection{Soluciones del problema}

Una vez representado un problema, éste puede ser resuelto algorítmicamente o no. En el primer caso la representación adecuada es el paso más importante, pues sólo queda aplicar los procedimientos. Si la representación no tiene solución algorítmica directa es fácil generar soluciones falsas y erradas; en este segundo caso el sujeto podrá resolverlo sí: a) detecta la no aplicabilidad de las rutinas que conoce; y b) desarrolla estrategias para localizar información pertinente o reconstruir lo que falta a partir de elementos asociados entre sí. Las estrategias son:

Generación y ensayo. Recorre la lista de elementos candidatos que se ajustan a un criterio, el cual define lo que hace falta hasta encontrar uno que los satisface.

Empleo de subobjetivos. Reformula objetivos parciales que avancen 1-n dirección del objetivo final y resuelve primero los parciales. Los expertos son más hábiles en esta estrategia: sus subobjetivos son resolubles. En esta estrategia las pistas que se ofrecen son entendidas en función de la representación que del subobjetivo como problema tiene el sujeto.

\section{PSICODIDÁCTICA NUMÉRICA}

La psicodidáctica numérica o algorítmica se orienta a la matemática tradicional y a sus componentes de cálculo, problemas y aplicaciones. Se divide generalmente en cursos de Aritmética, Algebra, Geometría y Cálculo. En la educación básica se orienta al dominio de las operaciones con números, sus mecanismos y las tablas correspondientes. Estudia y ejercita problemas modélicos de aplicación. Pueden distinguirse en esta psicodidáctica tres estrategias de enseñanza-aprendizaje: ejercicios de Thorndike, acumulaciones de Gagné y rutinas de Resnick. 


\subsection{Los ejercicios de Thorndike}

Se fundamenta en la ley del efecto descubierta por Thorndike en sus investigaciones sobre aprendizaje por ensayo y error, consistente en conexiones entre situación y respuesta. Si la conexión se acompaña de un estado satisfactorio aumenta su fuerza, si el estado es poco o nada satisfactorio entonces disminuye.

\subsubsection{Aplicación educativa}

El propósito pedagógico es establecer los vínculos y hábitos necesarios para el cálculo y los problemas. Debe analizarse los temas educativos para establecer el inventario de conexiones necesarias, cada una de las cuales debe recibir tratamiento pedagógico.

Las conexiones o vínculos pueden ser de dos tipos: propedéuticos y cooperativos. Las conexiones propedéuticas son utilizadas sólo para facilitar nuevos aprendizajes y deben ser temporales; por ejemplo, multiplicar 5 X 4, sumando $5,10,15,20$, que deberá ser sustituido por $5 \mathrm{X}$ $4=20$. Las conexiones cooperativas son vínculos organizados subyacentes a operaciones largas y complejas, que requieran para ser adquiridas una práctica progresiva y cuidadosa.

\subsubsection{Papel de la práctica}

Los vínculos se crean ofreciendo cantidades de ejercicios adecuados en orden apropiado para cada tipo de tema, progresando de lo sencillo a lo complejo. Buswell demostró que pequeñas cantidades de ejercicios frecuentes son mejores que largas concentraciones. Los ejercicios aislados pueden asentar lo recién aprendido y corregir respuestas equivocadas.

\subsubsection{Críticas al modelo}

Bronwell criticó en su momento a Thorndike por no tener en cuenta:

a) que hay diferencias cualitativas entre los cálculos del niño y los del adulto: el niño asume procedimientos propios; b) que el objetivo educativo es desarrollar la capacidad de pensar matemáticamente y no 
sólo resolver con precisión el 100\% de listas de problemas.

\subsection{Las acumulaciones de Gagné}

En el aprendizaje acumulativo de Gagné las tareas sencillas funcionan como componentes de las más complejas, permitiendo al aprender las sencillas transferir el aprendizaje a lo complejo. Las tareas se ordenan en una jerarquía de aprendizaje aplicable a todo conocimiento. Esta jerarquía abreviadamente consta de: a) resolución de problemas, que requieren como requisitos b) reglas, principios y conceptos definidos, que requiere c) conceptos concretos, que requiere d) discriminaciones, que, finalmente requiere e) conexiones E-R.

\subsubsection{Elaboración y validación de jerarquías}

Se realizan por un análisis racional de tareas; se identifican las destrezas y conocimientos esenciales, jerarquizados y configurados de arriba-abajo, necesarios para aprender una capacidad. Al análisis del primer nivel de prerrequisito y así sucesivamente. Se ignoran los requisitos más elementales. Hay que identificar claramente el objetivo final, el punto de partida y los pasos intermedios.

Las jerarquías se validan por los procedimientos: a) escalamiento, medido por el dominio de los prerrequisitos con los signos + y -. Luego se ordena a los estudiantes por rendimiento en la tarea. Debe generarse una escala Guttman; b) entrenamiento, ejercitado en los prerrequisitos él un grupo experimental, su rapidez y calidad de aprendizaje debe ser mejor que el del grupo control similar, pero sin entrenamiento.

\subsubsection{Aplicación educativa}

Hay dos condiciones para el éxito de la acción educativa: dominio de prerrequisitos y secuenciación de la enseñanza: a) dominio de prerrequisito: el aprendizaje solo puede iniciarse cuando se conoce las tareas previas que saben y dominan los educandos; b) secuenciación de la enseñanza: es la aplicación del ordenamiento de la enseñanza de la jerarquía de aprendizaje y el análisis de tareas. Las jerarquías 
específicas deben ser probadas empíricamente. Son un mapa para la secuencia de enseñanza.

\subsection{Rutinas de Resnick}

Esta psicodidáctica está centrada en los pasos mentales necesarios a dar para resolver una tarea: son las exigencias a la memoria y a las habilidades. La secuencia de pasos mentales es una rutina.

\subsubsection{Identificación de rutinas}

Son dos los procedimientos para identificar las rutinas: el análisis hipotético y el análisis de tarea:

a) El análisis hipotético es un análisis racional de los procesos y la elaboración de un diagrama de flujo. Ej.: para el conteo de objetos móviles que se pueden tocar y mover a voluntad hay el siguiente diagrama de flujo:

\begin{tabular}{|c|c|c|c|}
\hline $\mathrm{A}$ & B & $\mathrm{C}$ & D \\
\hline \begin{tabular}{l} 
Extraer un \\
objeto del \\
conjunto y decir \\
\multicolumn{2}{l}{ la cifra uno }
\end{tabular} & $\begin{array}{l}\text { Extraer un } \\
\text { objeto del } \\
\text { conjunto y decir } \\
\text { la cifra : dos, } \\
\text { tres, ...etc.. }\end{array}$ & $\begin{array}{l}\text { ¿Existen más } \\
\text { objetos en el } \\
\text { conjunto? } \\
\mathrm{Si} \quad \mathrm{No}\end{array}$ & $\begin{array}{l}\text { Declarar que la } \\
\text { última cifra } \\
\text { corresponde al } \\
\text { número del } \\
\text { conjunto }\end{array}$ \\
\hline
\end{tabular}

En esta tarea no se usa necesariamente la memoria (los objetos contados pueden ser separados). Si los objetos no pueden moverse hay una tarea adicional: recordar los objetos tocados pero no movidos.

b) El análisis de tareas se concreta en tres procedimientos básicos: tiempo de reacción, análisis de protocolos y problemas en contexto. En el análisis por tiempo de reacción hay que hallar los diferentes tiempos por tareas y por soluciones iguales con diferente estrategia. Por ejemplo, en suma hay tres posibles estrategias: contador se pone en cero, contador parte del primer número, y contador parte del número mayor. Los resultados demuestran la inversión de más tiempo con la primera estrategia y de 
menos tiempo con la tercera. En el análisis de protocolos se pretende identificar procedimientos y errores. Los protocolos son registros lo más completos posibles de lo que hace el sujeto al resolver un problema. Hay gran cantidad de estrategias poco ortodoxas. Los buenos matemáticos usan algoritmos, los malos cometen errores. Estos no son causales sino sistemáticos, derivados de su falta de comprensión. Finalmente el análisis por programas en contexto somete a comparación programas de computación con análisis de protocolos. Se ha descubierto que frente a los programas por ejemplo algebraicos (Bobrow) en los que la computadora primero divide el problema en frases, segundo traduce las frases a términos algebraicos, tercero escribe con ellos ecuaciones y las resuelve, el sujeto humano tiene dos variantes: una, el conocimiento amplio de equivalencias de frases; y otra, el uso de representaciones físicas de las que extrae información pertinente.

\subsubsection{Aplicaciones educativas}

La enseñanza debe orientarse al dominio de rutinas con comprensión de la naturaleza sistemática del algoritmo para su uso significativo. Debe superarse la incomprensión de los procedimientos y porque los errores no son casuales deben analizarse e identificarse las rutinas defectuosas usadas. La enseñanza debe ajustarse a las dificultades individuales y respetar las estrategias propias si son matemáticamente correctas y llevan a soluciones correctas. Debe insertarse en los siguientes aspectos educativos:

a) Doble codificación: tanto verbal como icónica, no sólo de problemas físicos sino de cantidades.bbb

b) Automatización: el dominio incompleto de las rutinas (ej. las tablas) genera mucho error por sobrecarga de la memoria en el procesamiento. Si se trabaja con currículo en espiral y no se registra en la memoria desde el inicio valores numéricos y algoritmos pertinentes y la práctica es escasa, habrán dificultades posteriores si ese dominio es incluido después como componente de tareas más complejas.

c) Transiciones en competencias: desde los procedimientos que utiliza el propio estudiante debe apoyarse mejores rutinas y estrategias, 
enseñándolas y fomentando la invención de rutinas más eficientes.

d) Conexión de relaciones: la memoria semántica no sólo debe asociar ítems sino relacionarlos o con más exactitud multirrelacionar los diversos componentes de dos o más redes semánticas entre sí.

e) Objetivos cognitivos: las redes semánticas deben concretizar objetivos cognitivos ampliando la estructura del conocimiento además del rendimiento o conducta. Los objetivos deben centrarse en conocimiento y procedimientos; para especificarlos deben tenerse en cuenta los siguientes aspectos: conceptos de la estructura del contenido, pautas bien estructuradas del conocimiento y por último, información empírica derivada de las estructuras del conocimiento de los expertos.

f) Enseñanza de problemas: debe ser un eje en la enseñanza cuidando, primero de dar conocimientos maximizando vínculos entre conocimientos bien estructurados y procedimientos; segundo tener en cuenta que los datos o entornos del problema son el estímulo primario para procesar la solución; tercero, diseñar estrategias concretas de solución de problemas, enseñando a anticipar y visualizar las posibles diversas vías de acción antes de definir y proceder por una de ellas.

\section{PSICODIDÁCTICA CONJUNTISTA}

La psicodidáctica conjuntista, estructural, lógica simbólica o algebraica está orientada al dominio de las estructuras algebraicas fundamentales de la matemática moderna. Sus partes son: el álgebra de conjuntos con temas como las operaciones entre conjuntos: disyunción, unión, etc., las relaciones entre elementos del conjunto, tales como: funciones, productos, y equivalencia; y las estructuras de los conjuntos tales como grupo, anillo, reticulado y sus propiedades de transformación y composición: los nuevos conceptos de la matemática moderna tienen un amplísimo rango de 
generalidad y un alto nivel de abstracción, estas propiedades le conceden mayor rigor lógico, un alto nivel de exigencia intutitiva y formalista y en consecuencia, según sus defensores, una mayor capacidad para reconocer por "tacto" lo relevante e importante matemáticamente. Sus detractores se basan en esas mismas características para criticar sus dificultades de aplicabilidad.

Son tres las psicodidácticas que se han orientado preferentemente a la matemática moderna: la comprensión de Gestalt, el descubrimiento de Bruner y el constructivismo de Dienes.

\subsection{La comprensión de la Gestalt}

Los conceptos fundamentales de la Gestalt con relevancia para las matemáticas son los de forma, insight y pensamiento productivo.

a) Forma (Wertheimer): el perceptor aporta a la percepción la configuración, que es más que la simple suma de elementos bajo las reglas de la pregnancia o buena forma.

b) Insight (Kohler): intelección súbita de una situación problema que reorganiza la situación en forma repentina y espontánea. Procede de la reestructuración de los elementos del problema que se ven en un nuevo contexto. Son famosos los experimentos realizados por Kohler con chimpancés.

c) Pensamiento productivo (Duncker): elaboración nueva, no reproductiva ante un problema. La solución productiva no es tan sólo un paso a partir del planteamiento del problema. Inicialmente surge el principio o valor funcional de la solución. Este principio se logra concretizar más y más. Las propiedades generales o esenciales de una solución son anteriores a las específicas: éstas se derivan de aquellas.

\subsubsection{Aplicaciones educativas}

a) Aprendizaje comprensivo. Orientar al sujeto al descubrimiento de los principios, estructura o relaciones subyacentes es mejor que un aprendizaje sin sentido: mejora la atención, la transferencia y la capacidad de reconstruir la solución. Katona esperimentó con números de trece cifras frente a las que los sujetos podían asumir tres tareas. 
Primera: repetir tres veces el número por grupo de tres cifras (centenas). Segundo: recordarlo como PBI del país. Tercera: aprenderla como una serie numérica. El mejor resultado a la semana, pues todos los grupos pudieron recitarla inmediatamente, fue el del grupo tercero que recordaba perfectamente la serie porque había descubierto la ley de su formación para aprenderla. El segundo grupo recordaba la cifra aproximada del valor PBI. El tercero no recordaba nada.

b) Facilitación del insight (Polya). Consiste en dar pistas y apoyos para descubrir las estructuras subyacentes cuando se trabaja un problema: son heurísticos orientados a cuatro estrategias secuenciales. Primera: comprender el problema, identificar datos e incógnita. Segundo: desarrollar un plan hacia la solución estableciendo las relaciones entre datos e incógnita usando problemas relacionados, replanteamiento, rodeos, etc. Tercero: ejecutar el plan comprobando la corrección de cada paso. Cuarto: examinar la solución obtenida y su congruencia con los datos y la incógnita.

\subsection{El descubrimiento de Bruner}

Las siguientes son las dos características esenciales del aprendizaje por descubrimiento: a) es producido por una indagación activa ante un problema cuya solución genera un conocimiento más profundo y completo, y b) debe reconocerse la situación y activarse los recursos cognitivos necesarios para procesar un producto que la satisfaga.

Según Glazer tiene características de aprendizaje inductivo por $\sim \mathrm{r}$ de los ejemplos a las reglas. También se ha señalado que es un aprendizaje con errores, porque el sujeto agrega su propia estructura a la secuencia instructiva propuesta. Debe orientársele a sacar provecho de sus errores y a reducir éstos al mínimo posible.

6.2.1 Principios del descubrimiento Son cuatro los principios propuestos:

a) Principio de sencillez: todo problema o cuerpo de conocimientos 
puede ofrecerse en forma sencilla como para que cualquier estudiante determinado 10 pueda comprender en forma reconocida.

b) Principio de la representación: el conocimiento de un campo se desarrolla ordenada y evolutivamente en una secuencia de formas representacionales: enactiva, icónica y simbólica, es decir en acción, imágenes y pensamiento verbal.

c) Principio del proceso: el descubrimiento estimula un modo de aprender operando y anima al desarrollo de una concepción matemática más como proceso que como producto.

d) Principio de la satisfacción: el descubrimiento es no intrínsecamente gratificante por lo que requieren formas extrínsecas de premio.

\subsubsection{Tipos de descubrimiento}

Bigg propuso cinco tipos de aprendizaje por descubrimiento. Reduciremos su propuesta a sólo tres tipos.

a) Fortuito y libre: es muy aleatorio y no garantiza aprendizaje.

b) Guiado: apoyado en función de la actuación del sujeto.

e) Dirigido y programado: es contradictorio a los mismos principios del descubrimiento, no se puede garantizar su eficacia.

El aprendizaje por descubrimiento no es absolutamente generalizable y exige altas condiciones profesionales y pedagógicas del profesor.

\subsection{El constructivismo de Dienes}

Está basado en los aportes psicoevolutivos de Piaget y de Bruner, y en una concepción estructuralista de la matemática moderna. 


\subsubsection{Principios fundamentales}

a) Principio dinámico: se supondrán primero juegos preliminares estructurados y prácticos, dan la experiencia indispensable. En los mayores puede hacerse juegos mentales.

b) Principio constructivo en la estructuración secuencia! de los juegos la construcción precederá siempre al análisis reflexivo. Este está generalmente ausente hasta los doce años.

c) Principio de variabilidad matemática: los conceptos con más de una variable deben ser estudiados con experiencias que supongan el manejo del mayor número posible de esas variables.

d) Principio de variabilidad perceptiva: es una exigencia de concretización múltiple, la misma estructura conceptual deberá presentarse en tantas formas perceptivas equivalentes como sea posible.

\subsubsection{Etapas didácticas}

La didáctica constructivista se ofrece en un modelo cíclico, es una secuencia en sus etapas, tres de ellas lúdicas y las otras tres representacionales.

Las primeras etapas son lúdicas. La primera es de libertad: manipulación libre de los materiales concretos y descubrimiento de sus propiedades. La segunda es de reglas: con los mismos materiales inventa o aplica reglas, debe hacérseles conscientes de los cambios de reglas. La tercera es de comparaciones: se comparan los juegos reglados par a par, encontrando semejanzas, diferencias, clases y tipos de juego.

Las últimas tres etapas son representacionales. La primera es espacial: realiza diagramas o diseños con la estructura común a los tipos de juego. La segunda es simbólica: determinación de un lenguaje para las propiedades comunes a los juegos. La tercera es formalizada: organización de un sistema ordenado de las propiedades de cada tipo de Juego. 


\section{BIBLIOGRAFÍA}

Baroody, A. (1994). El pensamiento matemático de los niños. Madrid: Visor. Brissiaud, E. (1993). El aprendizaje del cálculo. Madrid: Visor.

Deaño, D. (1993). Conocimiento lógico-matemático en la escuela infantiL. Madrid: CEPE.

Dicnes, Z. (1970). La construcción de las matemáticas. Barcelona: VicensVives.

Dienes, Z. (1971). El aprendizaje de la matemática. Buenos Aires: Estrada.

Diencs, Z. (1971). La potencia de la matemática. Buenos Aires: Estrada.

Dienes, Z. (1975). Enseñanza y aprendizaje de la matemática. Buenos Aires: Paidós.

Gagné, E. (1991). La psicología cognitivo del aprendizaje escolar. Madrid: Visor.

Gagné, E. (1987). Las condiciones del aprendizaje. México: Interamericana.

Holloway, G. (1969). Concepción de la geometría en el niño según Piaget. Buenos Aires: Paidós.

Holloway, G. (1969). Concepción del espacio en el niño según Piaget. Buenos Aires: Paidós.

Hughes, M. (1987). Los niños y los números. Barcelona: Planeta.

Inhelder, B. y Piaget, J. (1972). De la lógica del niño a la lógica del adolescente, Buenos Aires: Paidós ..

Issacs, N. (1967). Nueva luz sobre la idea de número en el niño. Buenos Aires: Paidós.

Jurado, C. (1993). Didáctica de la matemática. Quito: Abya- Vala.

Kamii, C. (1993). El niño reinventa la aritmética. Madrid: Visor.

Lave, J. (1991). La cognición en la práctica. Buenos Aires: Paidós.

Lawrence, E. y otros (1968). La comprensión del número y la educación del niño según Piaget. Buenos Aires: Paidós.

Lovell, K. (1969). Didáctica de las matemáticas. Madrid: Morata.

Maza, C. (1995). Aritmética y representación. Barcelona: Paidós.

Orton, A. (1990). Didáctica de las matemáticas. Madrid: Morata.

Piaget, J. Y Szeminska, (1967). Génesis del número en el niño. México: Guadalupe.

Piaget, J. y otros (1983). La enseñanza de las matemáticas modernas. Madrid: Alianza.

Rcsnick, L. Y Ford, W. (1990). La enseñanza de las matemáticas y sus fundamentos psicológicos. Barcelona: Paidós.

Resnick, L. Y Kloptcr, L. (1996). Currículum y cognición. Buenos Aires: 